\title{
ANTERIOR SPINAL ARTERY SYNDROME AND ITS NATURAL HISTORY
}

\author{
By Dominic Foo, M.D.,,$^{1,2,3}$ and Alain B. Rossier, M.D. ${ }^{1,4}$ \\ 1 Spinal Cord Injury and ${ }^{2}$ Neurology Services, West Roxbury Veterans Administration \\ Medical Center, and ${ }^{3}$ Department of Neurology and ${ }^{4}$ Orthopaedic Surgery, Harvard \\ Medical School, Boston, Mass., U.S.A.
}

\begin{abstract}
Summary. The anterior spinal artery syndrome in three patients is described and from the literature 60 additional patients were collected. Motor recovery in the following groups of patients was noted: (I) Partial loss of motor function and pain sensation-70.4 per cent (19/27); (2) Complete motor loss but partial loss of pain-83.3 per cent (5/6); (3) Paresis but pain sensation absent-66.7 per cent (6/9); and (4) Absent motor function and pain-38.9 per cent (7/I8). Motor recovery was also found to vary according to aetiology: (A) Unknown cause92.9 per cent (I3/I4); (B) Post-infection or vaccination-88.9 per cent (8/9); (C) Anterior spinal artery occlusion-33.3 per cent (3/9); (D) Spinal cord angioma20 per cent (2/IO); and (E) Aortic lesion-20 per cent (I/5).

Patients with sparing of motor function or pain sensation below the lesion do better than those without both functions. Neurological return also varies with the aetiology of the syndrome.
\end{abstract}

Key words: Anterior spinal artery (ASA); Motor function; Pain sensation.

\section{Introduction}

THE anterior spinal artery (ASA) syndrome was first described by Spiller (1909) in a patient with ASA thrombosis; and infarct was noted at autopsy in the anterior part of the spinal cord, extending from $\mathrm{C}_{4}$ to $\mathrm{T}_{3}$. Subsequently, it was found that this syndrome could also be the result of a fall in perfusion pressure (Garland et al., 1966), or local interference with the spinal cord blood supply (Mosberg et al., I 954; Weisman and Adams, I944). The necrosis at the site of infarct is less extensive than that seen in patients with anterior spinal cord injury (Foo et al., I982).

Here we report three patients. The literature was reviewed with regard to the relationship between the motor recovery and the initial neurological deficits, and between the motor recovery and the aetiology of the syndrome.

\section{Case Reports}

Patient I. A 57-year-old man presenting with clumsiness and weakness of his left hand, developed spasms and weakness with Renografin-6o. Intravenous Decadron, Mannitol and Phenobarbital were given, but in the ensuing 24 hours, he became tetraplegic with pain sensation absent but touch and proprioception preserved below $\mathrm{C}_{4}$ bilaterally.

There was some motor return in the left hand and legs before the patient was transferred to our centre four months after the onset of symptoms. Examination of the mental status and cranial nerves was normal. Except for some slight motion of the deltoid and biceps, the right upper limb was paralyzed; the left arm 
was severely paresed, with only slight voluntary deltoid, biceps, triceps and wrist flexion and extension power. Both upper extremities were flaccid and areflexic. The legs were weak, spastic and hyperreflexic with bilateral Babinski signs. Pain sensation was absent below $\mathrm{C}_{5}$ on the right and $\mathrm{C}_{4}$ on the left. Hypesthesia in C6-C8 on the right and from $\mathrm{C}_{4}$ to $\mathrm{T}_{\mathrm{I}}$ on the left was noted. Vibration was decreased at and below the shoulders but position sense was normal.

The patient showed no further motor improvement. Touch and vibration gradually returned to normal in the arms but pain sensation was unchanged. When he was discharged four months later, he was confined to a wheelchair.

Patient 2. A 27-year-old man was found, on radiological examination, to have an acute aneurysmal dilatation of the thoracic aorta after an automobile accident. He underwent surgical repair of the aorta on the same day. The thoracic aorta proximal to the left subclavian artery was found to be completely transsected; the only tissue holding the blood in place was the mediastinal pleura. The aorta was reconstructed with a Dacron graft. Cross clamping time of the aorta was about eight minutes. The patient was not hypotensive throughout the procedure. Immediately after the operation, he was noted to have flaccid paralysis of the legs with analgesia but normal proprioception below $\mathrm{T} 9$ on both sides. There was no neurological improvement after one year. (As far as the patient could recall there was no paralysis of the legs prior to the abdominal operations).

Patient 3. A 43-year-old man with a history of cervical spondylosis experienced severe chest and left arm pain when he was driving. Electrocardiogram was normal. Within 24 hours, the patient developed stiffness of the legs and paresthesia of the hands. Urinary retention occurred on the next day. Neurological examination two days after the onset showed paresis of the arms and the right leg and paralysis of the left leg. Pain sensation was impaired below T6 on both sides. Cervical pantopaque myelogram revealed enlargement of the spinal cord from $\mathrm{C}_{3}$ to $\mathrm{C} 6$ vertebral segments and multiple spondylotic bars from $\mathrm{C}_{3}$ to $\mathrm{C}_{7}$. Cerebrospinal fluid was clear with a protein level of $80 \mathrm{mg} / \mathrm{dl}$. Laminectomy from $\mathrm{C}_{3}$ to $\mathrm{C} 6$ was performed three days after the ictus. The meninges were opened and the dentate ligaments were cut. There was no epidural or subdural bleeding. The cervical spinal cord was flat anteroposteriorly.

There was some motor return in the legs after the operation. The patient was transferred to our centre six weeks post-laminectomy. Mental and cranial nerve functions were normal. Motor functions of deltoids and biceps were normal; other muscles in the upper and lower limbs were weak. The legs were spastic and hyperreflexic with bilateral Babinski signs. Biceps and brachioradialis reflexes were normal, but triceps reflexes hypoactive. Analgesia was present below T2 on the right and $\mathrm{T}_{4}$ on the left; touch and proprioception were normal.

After admission, further motor recovery was seen in all the extremities, but there was no return of pain sensation. Five months later, he was ambulating with a left short leg brace and two forearm crutches.

\section{Review of the Literature}

The literature of ASA syndrome with bilateral non-traumatic spinal cord involvement and dissociated sensory loss was reviewed. A total of 60 patients, 26 males and 34 females, with adequate clinical information were studied (Table I). The probable causes are set out in Table II.

The neurological information was obtained from the history and physical examination in the patient's first clinic visit. 'Paralysis' or 'paraplegia', in contrast to 'paresis' or 'paraparesis', was defined as 'a complete loss of motor power', unless the motor function of the individual 
TABLE I

Summary of 60 cases of anterior spinal artery syndrome collected from the literature

\begin{tabular}{|c|c|c|c|c|c|c|}
\hline \multirow[t]{2}{*}{ Author, Year } & \multirow{2}{*}{$\begin{array}{l}\text { Sex/ } \\
\text { Age } \\
(\mathrm{yr})\end{array}$} & \multirow{2}{*}{$\begin{array}{l}\text { Probable } \\
\text { cause }\end{array}$} & \multicolumn{3}{|c|}{ Neurological deficits } & \multirow{2}{*}{$\begin{array}{l}\text { Neurological } \\
\text { progress }\end{array}$} \\
\hline & & & Motor & Pain & Level & \\
\hline Albert, I969 & $\mathrm{M} / 50$ & Hypotension & Comp. & Comp. & $\mathrm{T}$ & No change \\
\hline Blankenhorn, I948 & $\mathrm{F} / 25$ & $\begin{array}{l}\text { Neo-ars- } \\
\text { phenamine }\end{array}$ & Incomp. & Comp. & $\mathrm{T}$ & $\begin{array}{l}\text { Major motor } \\
\text { recovery }\end{array}$ \\
\hline $\begin{array}{l}\text { Case 3801 } 2 \\
\quad(\mathrm{MGH}), \mathrm{I} 952\end{array}$ & $\mathrm{~F} / 64$ & $\begin{array}{l}\text { Anterior } \\
\text { spinal } \\
\text { artery } \\
\text { thrombosis }\end{array}$ & Comp. & Comp. & $\mathrm{L}$ & $\begin{array}{l}\text { No change, } \\
\text { death I } 2 \text { days } \\
\text { postictus }\end{array}$ \\
\hline CPC, I968 & $M / 66$ & $\begin{array}{l}\text { Cervical } \\
\text { spondylosis }\end{array}$ & Incomp. & Incomp. & $\mathrm{C}$ & $\begin{array}{l}\text { Some motor } \\
\text { recovery, death } \\
\text { I } 6 \text { days postictus }\end{array}$ \\
\hline Coupland, I968 & $\mathrm{M} / 59$ & $\begin{array}{l}\text { Abdominal } \\
\text { aortic } \\
\text { aneurectomy }\end{array}$ & Comp. & Comp. & $\mathrm{L}$ & No recovery \\
\hline \multirow[t]{2}{*}{ Deller, I960 } & $\mathrm{F} / 52$ & Unknown & Incomp. & Incomp. & $\mathrm{T}$ & $\begin{array}{l}\text { Major motor } \\
\text { recovery }\end{array}$ \\
\hline & $\mathrm{F} / 32$ & Unknown & Comp. & Incomp. & $\mathrm{T}$ & $\begin{array}{l}\text { Some motor } \\
\text { recovery }\end{array}$ \\
\hline Fiebrand, I966 & $\mathrm{M} / 4 \mathrm{I}$ & Unknown & Incomp. & Comp. & $\mathrm{C}$ & $\begin{array}{l}\text { Some motor } \\
\text { recovery }\end{array}$ \\
\hline \multirow[t]{2}{*}{ Garland, I966 } & $\mathrm{M} / 56$ & Hypotension & Incomp. & Comp. & $\mathrm{T}$ & $\begin{array}{l}\text { Major motor } \\
\text { recovery }\end{array}$ \\
\hline & $\mathrm{M} / 62$ & $\begin{array}{l}\text { Thoracic } \\
\text { aortic } \\
\text { aneurectomy }\end{array}$ & Incomp. & Comp. & $\mathrm{T}$ & $\begin{array}{l}\text { Some motor } \\
\text { recovery }\end{array}$ \\
\hline Grinker, I 927 & $\mathrm{M} / \mathrm{I} 5$ & $\begin{array}{l}\text { Anterior } \\
\text { spinal } \\
\text { artery } \\
\text { thrombosis }\end{array}$ & Comp. & Comp. & $\mathrm{C}$ & $\begin{array}{l}\text { No recovery, death } \\
6 \text { weeks postictus }\end{array}$ \\
\hline \multirow[t]{2}{*}{ Henneaux, I960 } & $\mathrm{F} / 35$ & $\begin{array}{l}\text { Anterior } \\
\text { spinal } \\
\text { artery } \\
\text { thrombosis }\end{array}$ & Incomp. & Incomp. & $\mathrm{C}$ & $\begin{array}{l}\text { Motor improve- } \\
\text { ment, death } 5 \\
\text { months postictus }\end{array}$ \\
\hline & $\mathrm{M} / 45$ & $\begin{array}{l}\text { Anterior } \\
\text { spinal } \\
\text { artery } \\
\text { thrombosis }\end{array}$ & Comp. & Comp. & $\mathrm{T}$ & $\begin{array}{l}\text { No recovery, } \\
\text { death } 5 \text { months } \\
\text { postictus }\end{array}$ \\
\hline Hogan, I942 & $\begin{array}{l}\text { M/un- } \\
\text { known }\end{array}$ & Unknown & Comp. & Comp. & $\mathrm{C}$ & $\begin{array}{l}\text { Motor improve- } \\
\text { ment }\end{array}$ \\
\hline Hogan, I966 & $\mathrm{M} / 76$ & $\begin{array}{l}\text { Abdominal } \\
\text { aortic } \\
\text { aneurectomy }\end{array}$ & Incomp. & Comp. & $\mathrm{L}$ & $\begin{array}{l}\text { No change, death } \\
24 \text { days postop. }\end{array}$ \\
\hline Hughes, 1964 & $\mathrm{~F} / 70$ & $\begin{array}{l}\text { Anterior } \\
\text { spinal } \\
\text { artery } \\
\text { thrombosis }\end{array}$ & Incomp. & Incomp. & $\mathrm{C}$ & $\begin{array}{l}\text { No recovery, death } \\
\text { I day later }\end{array}$ \\
\hline Lyon, I971 & $\mathrm{F} / 47$ & $\begin{array}{l}\text { Vertebral } \\
\text { angiogram }\end{array}$ & Incomp. & Incomp. & $\mathrm{C}$ & Major motor return \\
\hline Mosberg, I 954 & $\mathrm{~F} / 44$ & Sympathectomy & Comp. & Comp. & $\mathrm{T}$ & $\begin{array}{l}\text { Some motor } \\
\text { recovery }\end{array}$ \\
\hline & $\mathrm{F} / 35$ & Sympathectomy & Comp. & Comp. & $\mathrm{T}$ & No recovery \\
\hline Paine, I953 & $\begin{array}{l}\mathrm{M} / 5 \\
\mathrm{~F} / 9\end{array}$ & $\begin{array}{l}\text { Post-infection } \\
\text { Post-infection }\end{array}$ & $\begin{array}{l}\text { Incomp. } \\
\text { Incomp. }\end{array}$ & $\begin{array}{l}\text { Incomp. } \\
\text { Incomp. }\end{array}$ & $\begin{array}{l}\mathrm{L} \\
\mathrm{L}\end{array}$ & $\begin{array}{l}\text { Death within I week } \\
\text { Fair recovery }\end{array}$ \\
\hline
\end{tabular}


PARAPLEGIA

TABLE I (Continued)

\begin{tabular}{|c|c|c|c|c|c|c|}
\hline \multirow[t]{2}{*}{ Author, Year } & \multirow{2}{*}{$\begin{array}{l}\text { Sex/ } \\
\text { Age } \\
(y r)\end{array}$} & \multirow{2}{*}{$\begin{array}{l}\text { Probable } \\
\text { cause }\end{array}$} & \multicolumn{3}{|c|}{ Neurological deficits } & \multirow{2}{*}{$\begin{array}{l}\text { Neurological } \\
\text { progress }\end{array}$} \\
\hline & & & Motor & Pain & Level & \\
\hline & $\mathrm{F} / 5$ & Post-vaccination & Incomp. & Incomp. & L & Normal recovery \\
\hline & $\mathrm{F} / 5$ & Post-infection & Incomp. & Incomp. & $\mathrm{T}$ & Normal recovery \\
\hline & $\mathrm{F} / \mathbf{I O}$ & Post-infection & Incomp. & Incomp. & $\mathrm{L}$ & Good recovery \\
\hline & $\mathrm{M} / \mathbf{1} 5$ & Post-infection & Incomp. & Incomp. & $\mathrm{T}$ & Fair recovery \\
\hline & $\mathrm{F} / \mathrm{IO}$ & Unknown & Incomp. & Incomp. & $\mathrm{T}$ & Fair recovery \\
\hline & $\mathrm{F} / \mathrm{I} 2$ & Heavy lifting & Incomp. & Incomp. & $\mathrm{T}$ & Motor recovery \\
\hline & $\mathrm{M} / 5$ & Post-infection & Incomp. & Incomp. & $\mathrm{T}$ & Good recovery \\
\hline & $\mathrm{M} / \mathrm{I} 2$ & Unknown & Incomp. & Incomp. & $\mathrm{T}$ & Good recovery \\
\hline Parman, I980 & $M / 34$ & $\begin{array}{l}\text { Spinal epidural } \\
\text { bleeding }\end{array}$ & Comp. & Comp. & $\mathrm{T}$ & $\begin{array}{l}\text { Major motor } \\
\text { recovery postop. }\end{array}$ \\
\hline \multirow[t]{3}{*}{ Peterman, I958 } & $\mathrm{F} / 22$ & Unknown & Comp. & Comp. & $\mathrm{C}$ & $\begin{array}{l}\text { Motor improve- } \\
\text { ment }\end{array}$ \\
\hline & $\mathrm{F} / 54$ & Unknown & Comp. & Incomp. & $\mathrm{T}$ & $\begin{array}{l}\text { Motor improve- } \\
\text { ment }\end{array}$ \\
\hline & $\mathrm{F} / 49$ & Unknown & Incomp. & Comp. & $\mathrm{T}$ & $\begin{array}{l}\text { Motor improve- } \\
\text { ment }\end{array}$ \\
\hline \multirow[t]{2}{*}{ Schaller, I 932} & $\mathrm{~F} / 42$ & Unknown & Comp. & Comp. & C & Slight motor return \\
\hline & $\mathrm{F} / 42$ & Unknown & Comp. & Incomp. & $\mathrm{C}$ & $\begin{array}{l}\text { Motor improve- } \\
\text { ment }\end{array}$ \\
\hline Slavin, I 975 & $\mathrm{M} / 60$ & $\begin{array}{l}\text { Anterior } \\
\text { spinal } \\
\text { artery } \\
\text { emboli }\end{array}$ & Incomp. & Comp. & $\mathrm{L}$ & $\begin{array}{l}\text { Motor improve- } \\
\text { ment, death } 4 \\
\text { months postictus }\end{array}$ \\
\hline Spiller, I 909 & $\begin{array}{l}M / \text { un- } \\
\text { known }\end{array}$ & $\begin{array}{l}\text { Anterior } \\
\text { spinal } \\
\text { artery } \\
\text { thrombosis }\end{array}$ & Incomp. & Incomp. & $\mathrm{C}$ & $\begin{array}{l}\text { Motor improve- } \\
\text { ment }\end{array}$ \\
\hline \multirow[t]{5}{*}{ Steegman, I952 } & $\mathrm{F} / 38$ & Lues & Comp. & Comp. & $\mathrm{T}$ & Major motor return \\
\hline & $\mathrm{M} / 50$ & Lues & Incomp. & Incomp. & $\mathrm{T}$ & $\begin{array}{l}\text { Motor improve- } \\
\text { ment }\end{array}$ \\
\hline & $\mathrm{M} / 65$ & Post-infection & Incomp. & Incomp. & $\mathrm{C}$ & Some motor return \\
\hline & $\mathrm{F} / 60$ & Unknown & Comp. & Incomp. & $\mathrm{C}$ & $\begin{array}{l}\text { Motor improve- } \\
\text { ment }\end{array}$ \\
\hline & $\mathrm{F} / \mathrm{I} 4$ & Post-infection & Incomp. & Incomp. & $\mathrm{T}$ & Complete recovery \\
\hline Watson, I 967 & $\mathrm{~F} / 44$ & $\begin{array}{l}\text { Aortic } \\
\text { coarctation }\end{array}$ & Comp. & Comp. & $\mathrm{T}$ & Slight motor return \\
\hline Weisman, I 944 & $\mathrm{~F} / 68$ & $\begin{array}{l}\text { Dissecting } \\
\text { thoracic } \\
\text { aortic } \\
\text { aneurysm }\end{array}$ & Comp. & Comp. & $\mathrm{T}$ & $\begin{array}{l}\text { No recovery, } \\
\text { death } 5 \text { days } \\
\text { postictus }\end{array}$ \\
\hline \multirow[t]{5}{*}{ Wieringen, I 968} & $\mathrm{M} / 60$ & $\begin{array}{l}\text { Metastatic } \\
\text { spinal } \\
\text { carcinoma }\end{array}$ & Comp. & Comp. & $\mathrm{T}$ & $\begin{array}{l}\text { Some motor } \\
\text { recovery }\end{array}$ \\
\hline & $\mathrm{F} / 54$ & Unknown & Comp. & Incomp. & $\mathrm{T}$ & $\begin{array}{l}\text { Motor improve- } \\
\text { ment }\end{array}$ \\
\hline & $\mathrm{F} / 68$ & Unknown & Comp. & Comp. & $\mathrm{L}$ & $\begin{array}{l}\text { Motor improve- } \\
\text { ment }\end{array}$ \\
\hline & $\mathrm{F} / 66$ & $\begin{array}{l}\text { Metastatic } \\
\text { spinal } \\
\text { carcinoma }\end{array}$ & Comp. & Comp. & $\mathrm{T}$ & No recovery \\
\hline & $\mathbf{M} / 22$ & $\begin{array}{l}\text { Anterior } \\
\text { spinal } \\
\text { artery } \\
\text { occlusion }\end{array}$ & Comp. & Incomp. & $\mathrm{C}$ & $\begin{array}{l}\text { Slight motor } \\
\text { recovery }\end{array}$ \\
\hline
\end{tabular}


TABLE I (Continued)

\begin{tabular}{|c|c|c|c|c|c|c|}
\hline \multirow[t]{2}{*}{ Author, Year } & \multirow{2}{*}{$\begin{array}{l}\text { Sex/ } \\
\text { Age } \\
(y r)\end{array}$} & \multirow{2}{*}{$\begin{array}{l}\text { Probable } \\
\text { cause }\end{array}$} & \multicolumn{3}{|c|}{ Neurological deficits } & \multirow{2}{*}{$\begin{array}{l}\text { Neurological } \\
\text { progress }\end{array}$} \\
\hline & & & Motor & Pain & Level & \\
\hline \multirow[t]{10}{*}{$\begin{array}{l}\text { Wyburn-Mason, } \\
\text { I } 943\end{array}$} & $\mathrm{M} / 25$ & $\begin{array}{l}\text { Spinal cord } \\
\text { angioma }\end{array}$ & Incomp. & Incomp. & $\mathrm{T}$ & $\begin{array}{l}\text { Recurrence of } \\
\text { symptoms }\end{array}$ \\
\hline & $\mathrm{M} / 40$ & $\begin{array}{l}\text { Spinal cord } \\
\text { angioma }\end{array}$ & Incomp. & Incomp. & $\mathrm{T}$ & $\begin{array}{l}\text { Recurrence of } \\
\text { symptoms }\end{array}$ \\
\hline & $\mathrm{F} / 34$ & $\begin{array}{l}\text { Spinal cord } \\
\text { angioma }\end{array}$ & Incomp. & Incomp. & $\mathrm{T}$ & $\begin{array}{l}\text { Major motor } \\
\text { recovery }\end{array}$ \\
\hline & $\mathrm{M} / 54$ & $\begin{array}{l}\text { Spinal cord } \\
\text { angioma }\end{array}$ & Incomp. & Incomp. & $\mathrm{L}$ & No recovery \\
\hline & $\mathrm{F} / 40$ & $\begin{array}{l}\text { Spinal cord } \\
\text { angioma }\end{array}$ & Incomp. & Comp. & $\mathrm{T}$ & No recovery \\
\hline & $\mathrm{F} / 47$ & $\begin{array}{l}\text { Spinal cord } \\
\text { angioma }\end{array}$ & Incomp. & Comp. & $\mathrm{T}$ & No recovery \\
\hline & $\mathrm{F} / 49$ & $\begin{array}{l}\text { Spinal cord } \\
\text { angioma }\end{array}$ & Incomp. & Incomp. & $\mathrm{L}$ & No recovery \\
\hline & $\mathrm{F} / \mathrm{I} 7$ & $\begin{array}{l}\text { Spinal cord } \\
\text { angioma }\end{array}$ & Incomp. & Incomp. & $\mathrm{C}$ & $\begin{array}{l}\text { Motor improve- } \\
\text { ment }\end{array}$ \\
\hline & $\mathrm{M} / \mathrm{I} 7$ & $\begin{array}{l}\text { Spinal cord } \\
\text { angioma }\end{array}$ & Incomp. & Incomp. & $\mathrm{T}$ & $\begin{array}{l}\text { Recurrence of } \\
\text { symptoms }\end{array}$ \\
\hline & $\mathrm{M} / 47$ & $\begin{array}{l}\text { Spinal cord } \\
\text { angioma }\end{array}$ & Incomp. & Incomp. & $\mathrm{L}$ & No recovery \\
\hline Zeitlin, 1936 & $\mathrm{~F} / 45$ & $\begin{array}{l}\text { Anterior } \\
\text { spinal } \\
\text { artery } \\
\text { atheroma }\end{array}$ & Comp. & Comp. & $\mathrm{T}$ & $\begin{array}{l}\text { No recovery, } \\
\text { death I } 2 \text { weeks } \\
\text { postictus }\end{array}$ \\
\hline
\end{tabular}

Comp. = Complete; Incomp. = Incomplete; postop. = postoperatively; $\mathrm{C}=$ Cervical; $\mathrm{T}=$ Thoracic; $\mathrm{L}=$ Lumbar.

TABLE II

Probable causes of anterior spinal artery syndrome in 60 patients

Probable cause

Number of patients

Unknown

Spinal cord angioma

Post-infection or vaccination

Occlusive lesion of anterior spinal artery

Aortic disorder

Dorsolumbar sympathectomy

Hypotension

Lues without autopsy of spinal cord

Metastatic spinal carcinoma

Cervical spondylosis without anterior spinal artery thrombosis

Vertebral angiogram

Spontaneous spinal epidural bleeding

Intravenous neo-arsphenamine

Physical exertion 
muscle was given in the case record. Similarly, 'impairment' meant 'a reduction of strength or quantity'. 'Motor recovery or improvement' was considered as 'more than slight or minimal motor return'. If the neurological symptoms recurred or became worse, or if the patient expired without any description of his/her progress, he/she was assumed to have made no recovery.

Table III shows the presence of varying degree of motor return in the patients. The mortality is analysed in Table IV.

In Table $\mathrm{V}$, the prognosis in relation to the aetiology of the syndrome is shown. The outlook for recovery appeared to be more favourable in the patients with an unknown cause (92.9 per cent or 13/14) or postinfectious myelopathy (88.9 per cent of $8 / 9)$ than in those with an ASA occlusive lesion (33.3 per cent or 3/9), spinal cord angioma (20 per cent or $2 / \mathrm{IO}$ ) or aortic disorder ( 20 per cent or $\mathrm{I} / 5$ ). The absence of both motor function and pain sensation was noted in 28.6 per cent (4/I4), o per cent,

\section{TABLE III}

Motor recovery in anterior spinal artery syndrome in 60 patients

\begin{tabular}{|c|c|c|}
\hline Neurological deficits & Total number & Patients with motor recovery \\
\hline $\begin{array}{l}\text { Incomplete loss of motor } \\
\text { function and pain sensation }\end{array}$ & 27 & I9 $\left(70 \cdot 4_{0}^{\circ}\right)$ \\
\hline $\begin{array}{l}\text { Complete motor loss but } \\
\text { partial loss of pain }\end{array}$ & 6 & $5\left(83 \cdot 3^{\circ} \cdot 0\right)$ \\
\hline $\begin{array}{l}\text { Partial motor loss but } \\
\text { absent pain sensation }\end{array}$ & 9 & $6\left(66 \cdot 7_{0}^{\circ}\right)$ \\
\hline $\begin{array}{l}\text { Absent motor function } \\
\text { and pain sensation }\end{array}$ & I 8 & $7\left(38 \cdot 9^{\circ} \circ\right)$ \\
\hline Total & 60 & $37\left(\right.$ mean, $\left.6 \mathrm{I} \cdot 7^{\circ} \%\right)$ \\
\hline
\end{tabular}

TABLE IV

Mortality in anterior spinal artery syndrome in 60 patients

\begin{tabular}{|c|c|c|}
\hline Neurological deficits & Total number & $\begin{array}{l}\text { Mortality within } 6 \text { months } \\
\text { of the onset of symptoms }\end{array}$ \\
\hline $\begin{array}{l}\text { Incomplete loss of motor } \\
\text { function and pain sensation }\end{array}$ & 27 & $4(14.8 \%)$ \\
\hline $\begin{array}{l}\text { Complete motor loss but } \\
\text { partial loss of pain }\end{array}$ & 6 & o \\
\hline $\begin{array}{l}\text { Partial motor loss but } \\
\text { absent pain sensation }\end{array}$ & 9 & $2(22 \cdot 2 \%)$ \\
\hline $\begin{array}{l}\text { Absent motor function } \\
\text { and pain sensation }\end{array}$ & I 8 & $5\left(27 \cdot 8^{\circ}{ }_{0}\right)$ \\
\hline Total & 60 & I I (mean, I $\left.8 \cdot 3^{\circ}{ }_{0}\right)$ \\
\hline
\end{tabular}


TABLE V

Motor recovery in relation to the aetiology of anterior spinal artery syndrome in 47 patients

\begin{tabular}{|c|c|c|}
\hline Cause & $\begin{array}{l}\text { Patients with } \\
\text { motor recovery }\end{array}$ & $\begin{array}{l}\text { Patients without motor } \\
\text { function and pain sensation }\end{array}$ \\
\hline Unknown ( $\mathrm{N}=\mathrm{I} 4)$ & I3 $(92 \cdot 9 \%)$ & $4(28 \cdot 6 \%)$ \\
\hline $\begin{array}{l}\text { Post-infection or } \\
\text { vaccination }(\mathrm{N}=9)\end{array}$ & $8\left(88 \cdot 9^{\circ}\right)$ & o \\
\hline $\begin{array}{l}\text { Anterior spinal artery } \\
\text { occlusive lesion }^{\star}(N=9)\end{array}$ & $3\left(33 \cdot 3^{\circ} \circ\right)$ & $4(44 \cdot 4 \%)$ \\
\hline Spinal cord angioma $(\mathrm{N}=\mathrm{IO})$ & $2\left(20^{\circ}\right)$ & o \\
\hline Aortic disorder $(\mathrm{N}=5)$ & I $\left(20^{\circ}\right)$ & $3\left(60^{\circ}, 0\right)$ \\
\hline
\end{tabular}

$\star$ Diagnosed at autopsy.

44.4 per cent $(4 / 9)$, o per cent and 60 per cent $(3 / 5)$ of these five groups of patients, respectively.

\section{Discussion}

The clinical picture of ASA syndrome varies with the level of ischaemia. There is different degree of muscle weakness and cissociated sensory loss: pain sensation is decreased or absent while proprioception is relatively or completely spared. The loss of motor power usually parallels that of pain because of the anatomical proximity of the pyramidal and spinothalamic tracts in the cord. Rarely, this syndrome can also be the result of a cental spinal cord infarct (Hogan and Romanul, I966).

Lues was at one time a common cause of ASA syndrome (Henneaux, I960; Spiller, I909; Steegmann, I952). In children, it is often associated with post-infectious or post-vaccination myelopathy (Paine and Byers, I953). In adults, spinal cord angioma or vascular malformation is probably the most common aetiology (Hughes, I978; Wyburn-Mason, I943). Myelopathy was occasionally the result of angiography of the aorta, vertebral artery, or spinal cord but the frequency of this complication has been greatly reduced since the introduction of less toxic contrast material (Killen and Foster, I966). Patient I developed neurological symptoms when he was undergoing a transfemoral vertebral angiogram with Renografin-6o. Myelopathy following catheter vertebral angiography has been described, but Renografin-60 was implicated in only one patient in the literature (Lyon, I97I). Patient 2 was found to have a thoracic aortic aneurysm after an automobile accident but he did not become paralysed until after surgical intervention; hypotension was absent and cross clamping time of the aorta was not prolonged. Spinal cord infarct secondary to post-traumatic aortic aneurysms has not been reported (Mulder and Groliman, I969; Parmley et al., I 958; Strassman, I947). This is probably because most of these patients die shortly after injury without autopsy of 
the spinal cord and, in those who survive and develop a chronic aortic aneurysm, there is no interference with the spinal cord circulation. On the other hand, myelopathy sometimes follows aortic surgery (Garland et al., 1966; Hogan and Romanul, 1966). In Patient 2, traumatic dissection of an anterior medullary artery might have occurred and the spinal cord blood supply was probably further reduced during operation. Cervical spondylosis is rarely complicated by ASA syndrome: at autopsy, a patient was noted to have ASA thrombosis (Hughes and Brownell, I964), but another patient had a patent ASA (Clinicopathological Conference, I968). In these two patients the authors suggested that minor neck movements might have been the precipitating factor. Patient 3, who had cervical spondylosis, developed symptoms while driving and there was no history of neck movement. The causal relationship between cervical spondylosis and ASA syndrome requires further investigation.

About 60 per cent of the patients with ASA syndrome regained some motor function after several days (Table III), but complete motor return was unusual (Table I). The patients with some preservation of motor function or pain sensation below the level of lesion progressed better than those without these two functions (Tables III and IV). Similar to the completely paralysed patients with anterior spinal cord injury (Foo et al., I98I), the prognosis was worse when there was no sparing of pain sensation (Table III). The loss of motor or pain function associated with spinal cord angiomas is usually incomplete (Wyburn-Mason, I943), but the neurological deficits tend to recur and total excision of the lesion is difficult. The outlook for recovery was more favourable in patients with an unknown aetiology or post-infectious myelopathy than in those with an ASA occlusive disease or aortic disorder; this correlated with the severity of their neurological deficits (Table V).

\section{RÉSUMÉ}

Les auteurs décrivent 3 patients avec un syndrome de l'artère spinale antérieure et revoient les 60 cas publiés dans la littérature. Une amélioration motrice se manifesta dans les groupes de patients suivants, ceux avec: (I) perte partielle de la motricité et de la sensibilité douloureuse $-70,4 \%(19 / 27) ;(2)$ perte complète de la motricité avec préservation partielle de la sensibilité douloureuse $-83,3^{\circ}{ }_{0}(5 / 6) ;(3)$ parésie mais analgésie $-66,7 \%(6 / 9)$; et (4) perte complète de la motricité et de la sensibilité douloureuse $-38,9 \%(7 / 18)$. La récupération motrice se trouva aussi varier selon l'étiologie du syndrome: (a) étiologie inconnue $-92,9 \%$ (I $3 / 14)$; (b) post-infectieuse ou post-vaccinale $-88,9 \%(8 / 9)$; (c) occlusion de l'artère spinale anterieure $-33,3 \%(3 / 9 ;$ (d) angiome médullaire $-20 \%(2 /$ ro $)$; et (e) lésion aortique $-20 \%(\mathrm{I} / 5)$. L'analyse de l'ensemble des cas révèle que les patients avec préservation sous-lesionnelle de la motricité ou de la sensibilité douloureuse ont un meilleur pronostic que ceux sans ces deux modalités. La récupération neurologique dépend aussi de l'étiologie du syndrome.

\section{ZUSAMMENFASSUNG}

Die Autoren beschreiben 3 Patienten mit Spinalis-anterior-Syndrom. Sechzig Fälle wurden von der Literatur zusammenhergestellt. Eine motorische Wiederherstellung wurde in den folgenden Gruppen von Patienten beobachtet: (I) unvollständige Lähmung und inkomplette Schmerzausfall $-70,4 \%$ (19/27); (2) motorisch komplette Ausfälle aber partielle Analgesie $-83,3 \%(5 / 6)$; (3) Paresie aber vollständige Analgesie $-66,7 \%(6 / 9)$; und (4) vollständige Lähmung und Analgesie -38,9\%(7/18). Die motorische Wiederherstellung änderte sich im Bezug auf die Aetiologie des Syndroms: (a) unbekannt $-20 \%(\mathrm{I} / 5)$; (b) 
post-infektiös oder-vakzinal $-88,9^{\circ}{ }_{0}(8 / 9)$; (c) Verschluss der vorderen Spinalarterie $-33,3^{\circ}{ }_{0}(3 / 9)$; (d) Angiome medullaris $-20^{\circ}{ }_{0}(2 / 10)$; und (e) Aortaläsion $-20^{\circ}{ }_{0}(\mathrm{I} / 5)$. Es wurde gezeigt, dass Patienten mit motorischer Aussparung oder erhaltener Schmerzempfindung unterhalb der Läsion besser machen als die jenige ohne beide Funktionen. Neurologische Rückbildungerscheinungen hängen von der Aetiologie des Syndroms ab.

This paper was presented at the 106th Annual Meeting of the American Neurological Association in San Francisco, California, U.S.A., I4-16 September I981, and was supported in part by the New England Paralyzed Veterans of America. Address reprint requests to Dr Foo, West Roxbury Veterans Administration Medical Center, I400 VFW Parkway, Boston, MA 02132 , U.S.A.

\section{REFERENCES}

Albert, M. L., Greer, W. E. R., Kantrowitz, W. (I969). Paraplegia secondary to hypotension and cardiac arrest in a patient who has had previous thoracic surgery. Neurology $(N y), 19,915-918$.

BlankenhorN, D., WolfF, H. G. (1948). Myelitis following the administration of neoarsphenamine. F. Lab. Clin. Med., 33, I I65-1 168.

Case Records (CAse 38012) OF the Massachusetts General Hospital (1952). Anterior spinal artery thrombosis. New Engl. F. Med., 246, 30-34.

CLINICOPATHOLOGIC CONFERENCE (I968). A sixty-six year old man with the sudden onset of left-sided chest and arm pain, weakness of the extremities and urinary retention. Am. F. Med., 44, 430-440.

Coupland, G. A. E., ReEve, T. S. ( 1968). Paraplegia: a complication of excision of abdominal aortic aneurysm. Surgery, 64, 878-88I.

Deller, J. J. JR., Scalettar, R., Levens, A. J. (I960). Pain as a manifestation of acute anterior-spinal-artery thrombosis. New Engl. F. Med., 262, 1078-1079.

Fiebrand, H. (1966). Zur Pathogenese des Spinalis-anterior-Syndroms. Schweiz. Arch. Neurol. Neurochir. und Psychiat., 97, 227-233.

Foo, D., Subrahmanyan, T. S., Rossier, A. B. (I98I). Post-traumatic acute anterior spinal cord syndrome. Paraplegia, 19, 20I-205.

Foo, D., Bignami, A., Rossier, A. B. (I982). Post-traumatic anterior spinal cord syndrome: pathological studies of two patients. Surg. Neurol., 17, 370-375.

Garland, H., GreenberG, J., Harriman, D. G. F. (I966). Infarction of the spinal cord. Brain, 89, 645-662.

GrINKER, R. R., GUY, C. C. (1927). Sprain of cervical spine causing thrombosis of anterior spinal artery. F. A.M. A., 88, I I40-1 I 42 .

Henneaux, J. (I960). Étude clinique et anatomo-pathologique de deux cas de thrombose de l'artère spinale antérieure. Rev. Neurol., 102, 44-60.

Hogan, B. W. (1942). Acute myelitis: syndrome of occlusion of the anterior spinal artery at the first thoracic cord segment with softening of the cord. $U$. S. Navy Med. Bull., 40, I75-178.

Hogan, E. L., Romanul, F. C. A. (1966). Spinal cord infarction occurring during insertion of aortic graft. Neurology $(N y), \mathbf{1 6}, 67-74$.

Hughes, J. T. (1978). Pathology of the Spinal Cord, 2nd ed. Lloyd-Luke, London.

Hughes, J. T., Brownell, B. (I964). Cervical spondylosis complicated by anterior spinal artery thrombosis. Neurology $(N y)$, 14, 1073-1077.

Killen, D. A., Foster, J. H. (I966). Spinal cord injury as a complication of contrast angiography. Surgery, 59, 969-98I.

Lyon, L. W. (I97I). Transfemoral vertebral angiography as a cause of anterior spinal artery syndrome: case report. F. Neurosurg., 35, 328-330.

Mosberg, W. H. JR., Voris, H. C., Duffy, J. (I954). Paraplegia as a complication of sympathectomy for hypertension. Ann. Surg., 139, 330-334.

Mulder, D. G., Grollman, J. H. (1969). Traumatic disruption of the thoracic aorta: diagnostic and surgical considerations. Am. F. Surg., I I8, 3 I I-3 I6.

Paine, R. S., Byers, R. K. (I953). Transverse myelopathy in childhood. Am. F. Dis. Child., 85, I $5 \mathrm{I}-\mathrm{I} 63$.

Parman, S. C. (I980). Spontaneous spinal epidural hematoma: case report. Ann. Emerg. Med., 9, 368-370. 
Parmley, L. F., Mattingly, T. W., Manion, W. C., Jahnke, E. J. JR. (I958). Non-penetrating traumatic injury of the aorta. Circulation, I7, I086-I IOI.

Peterman, A. F., Yoss, R. E., Corbin, K. B. (1958). The syndrome of occlusion of the anterior spinal artery. Proc. Mayo Clinic, 33, 3I-37.

Schaller, W. F., Roberts, A. M., StadTherR, E. F. (I932). Acute myelitis (myelomalacia): syndrome of occlusion of the anterior spinal artery at the fifth cervical cord segment. F. $A$. M. A., 99, I 572-1575.

Slavin, R. E., Gonzalez-Vitale, J. C., Marin, O. S. M. (1975). Atheromatous emboli to the lumbosacral spinal cord. Stroke, 6, 4I I-4I6.

SPILlER, W. G. (I909). Thrombosis of the cervical anterior median spinal artery; syphilitic acute anterior poliomyelitis. F. Nerv. E Ment. Dis., 36, 60I-6I3.

SteEgmanN, A. T. (1952). Syndrome of the anterior spinal artery. Neurology $(N y)$, 2, I 5-35.

Strassman, G. (1947). Traumatic rupture of the aorta. Am. Heart f., 33, 508-51 5.

WATSON, A. B. (1967). Spinal subarachnoid haemorrhage in patient with coarctation of aorta. Br. Med. F., 4, 278-279.

Weisman, A. D., AdAmS, R. D. (I944). The neurological complications of dissecting aortic aneurysm. Brain, 67, 69-92.

WIERINGEN, A. V. (I968). An unusual cause of occlusion of the anterior spinal artery. Europ. Neurol., I, 363-374.

WyBURN-MASON, R. (I943). Vascular Abnormalities and Tumours of Spinal Cord and Its Membranes. Henry Kimpton, London.

Zeitlin, H., Lichtenstein, B. W. (I936). Occlusion of the anterior spinal artery: clinicopathologic report of a case and a review of the literature. Arch. Neurol. Psychiat., 36, 96-I I I. 